\title{
Launch of an Open Access peer-reviewed journal on the treatment of respiratory diseases with integrated traditional Chinese and Western medicine
}

\author{
Xianmei Zhou ${ }^{1,2,3, *}$ \\ ${ }^{1}$ Department of Respiratory and Critical Care Medicine, the Affiliated Hospital of Nanjing University of Chinese Medicine, \\ Nanjing 210029, China \\ ${ }^{2}$ Department of Integrated Chinese and Western Medicine, the First School of Clinical Medicine, \\ Nanjing University of Chinese Medicine, Nanjing 210029, China \\ ${ }^{3}$ Inaugural Editor in Chief of Integrative Respiratory Medicine (IRM)
}

Received 17 August 2020, Accepted 17 August 2020

\begin{abstract}
We launch "Integrative Respiratory Medicine (IRM)" a new peer-reviewed Open Access journal treating the progress in diagnostic methods as well as comprehensive treatment of integrated traditional Chinese and Western medicine on respiratory diseases. The editorial team believes that IRM will receive a wide readership and soon become a recognized source of innovative and exciting papers in the integrative respiratory medical disciplines.
\end{abstract}

Respiratory diseases need multidisciplinary thoughts and measures. More than ever, this need for multidisciplinary and combination of different types of medicine was and is put in evidence with the outbreak and spreading of COVID-19.

Being the Head of the Department of Integrated Chinese and Western Medicine, the First School of Clinical Medicine, Nanjing University of Chinese Medicine, I realize the necessity of reporting and discussing at the academic level the progress in diagnostic methods as well as comprehensive treatment of integrated traditional Chinese and Western medicine on respiratory diseases.

We launch "Integrative Respiratory Medicine (IRM)": a journal treating exactly the above questions with the aim that this discussion and exchange be open and available for every reader, worldwide and so, the authors have a high visibility of their published work. Thus, the journal will be published in Open Access with the STM publisher EDP Sciences (actively supporting Open Access and publishing many Open Access journals, books and conference proceedings).

IRM will be driven by a board of high level researchers and an experienced Editorial Team. The journal is aimed to conduct a rigorous peer-review according to the quality standards of International Scientific Journals, for all the submitted manuscripts. The language of the journal is English and IRM welcomes papers of international research collaborations and papers from all over the world. IRM is aimed at promoting scientific exchanges between China and other parts of the world.

All the papers submitted to the journal will be structured, focused and highly relevant to integrative respiratory medicine.

It is required that all manuscripts submitted for publication in IRM contain new insight; present the results against a properly referenced background of existing work; present adequate evidence that supports the conclusions; written clearly concisely and comprehensively in English and include all necessary and appropriate figures and tables.

The types of publications considered are editorials, review articles, original research articles, case reports, methodologies, letters to the editor, systematic review, and short communications relating to integrative respiratory medicine.

The editorial team believes that IRM will receive a wide readership and soon become a recognized source of innovative and exciting papers in the integrative respiratory medical disciplines.

Cite this article as: X. Zhou 2020. Launch of an Open Access peer-reviewed journal on the treatment of respiratory diseases with integrated traditional Chinese and Western medicine. Integrat Respir Med, 1, E1.

*Corresponding author: zhouxianmeijs@aliyun.com

This is an Open Access article distributed under the terms of the Creative Commons Attribution License (https://creativecommons.org/licenses/by/4.0), which permits unrestricted use, distribution, and reproduction in any medium, provided the original work is properly cited. 